\title{
Phylogenetic analysis of a retroposon family as represented on the human $X$ chromosome
}

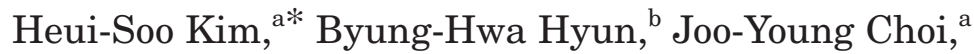 \\ and Timothy J. Crow ${ }^{\mathrm{c}}$ \\ ${ }^{a}$ Division of Biological Sciences, College of Natural Sciences, Pusan National University, Pusan, Korea \\ ${ }^{\mathrm{b}}$ Genetic Resources Center, Korea Research Institute of Bioscience and Biotechnology, \\ Taejeon, Korea \\ ${ }^{\mathrm{c}}$ POWIC, Department of Psychiatry, Warneford Hospital, University of Oxford, \\ Oxford, United Kingdom
}

(Received 1 February 2000, accepted 28 August 2000)

\begin{abstract}
SINE-R elements constitute a class of retroposons derived from the long terminal repeat (LTR) of the human endogenous retrovirus HERV-K family that are present in hominoid primates and active in the human genome. In an investigation of the $\mathrm{X}$ chromosome, we identified twenty-five SINE-R elements with between 89.6 and 97.7\% homology with the SINE-R.C2 element that is human specific, originally identified in the gene for the C2 component of complement. SINE-R.C2 and a sequence HS307 that we previously identified in a region of Xq21.3 that has a recently created homology with a $4 \mathrm{Mb}$ block in Yp11.2 are amongst the group of elements that have diverged furthest from the parent HERV-K10 sequence. The sequences on the $\mathrm{X}$ chromosome resemble those that we previously described on chromosomes 7 and 17 and the $\mathrm{Y}$ chromosome, with a similar range of variation. Phylogenetic analysis from the retroposon family including those of African great apes using the neighbor-joining method suggests that the SINE-R retroposon family have evolved independently during primate evolution. Further investigation of SINE-R elements on the sex chromosomes, particularly in recently created regions of X-Y homology, may cast light on the timing of the retroposition process and its possible relevance to recent evolutionary change.
\end{abstract}

\section{INTRODUCTION}

Mobile elements that transpose through reverse transcription are potential agents of genome change (Baltimore, 1985; Brosius and Tiedge, 1995; Kazazian and Moran, 1998; Sverdlov, 1998). However as much as one third of the mammalian genome is comprised of repetitive sequences of which the two major families of LINEs and SINEs are represented in humans by $\mathrm{L} 1$ and Alu elements respectively (Smit, 1996; Miki, 1998). SINE elements have generally arisen either from 7SL RNA or from tRNA, have been amplified many times in the genome, and have become fixed at certain stages of evolution. They share common 3' sequences with LINE elements which probably have an earlier evolutionary origin and upon which they may depend for the reverse transcriptase acivity necessary for retroposition (Okada et al., 1997). It must be assumed that in general both types of element have spread by a process of neutral evolution (Okada, 1991), and the over-

Edited by Naruya Saitou

* Corresponding author. E-mail: khs307@hyowon.cc.pusan.ac.kr whelming majority of such sequences must be inactive and have no functional consequence in terms of the organisms adaptation.

However particular interest attaches to the possibility that some such retropositional events are associated with events that have evolutionary significance. There have been suggestions that the process of speciation is related to the movement of genetic elements within the genome (Rose and Doolittle, 1983; Ginzburg et al., 1984), perhaps associated with chromosomal change (McClintock, 1984). The difficulty in investigating such a possibility is in developing a strategy to distinguish what might be recent and critical in species change from the long-standing background of retropositional activity in the mammalian genome. One approach to the problem is through the phylogenies of retroposons themselves. It has been suggested for example that whereas there is a relationship between LINEs and SINEs, specifically that SINEs are dependent upon the retropositional activity of an associated LINE element (Okada et al., 1997), the former have a much longer evolutionary history than the latter and that classes of SINEs can be identified that are restricted to 
certain groups of species (Terai et al., 1998). Thus the CHR1 SINEs are distributed in cetaceans, hippopotamus and ruminants but not in other mammals (Shimamura et al., 1997) and Hpa1 SINEs are distributed specifically in the Salmonidae but not in other fishes (Kido et al., 1991), the SmaI family is confined to the chum and pink salmon, and the DANA element is described as present only in the zebrafish (Izvak et al., 1996).

Thus the history of SINE elements in human evolution and their relationship to other transposable elements acquires particular interest. Are there specific sequences that are evolving in the primate and hominoid lineages, and if so which changes are most recent? What relationship might such changes have to other e.g. chromosomal changes in the genome? We have reported that the SINER.C2, a human specific retroposon (Zhu et al., 1994) that was derived from the HERV-K sequence, has close homologies with two sequences (HS307 and HS408) within the Xq21.3 block of that transposed to the Y chromosome sometime after the separation of the human and chimpanzee lineages (Kim et al., 1999b), and that a number of similar elements are present on the 7, 17, and Y chromosomes (Kim and Crow, 1999, 2000) and in the African great apes (Kim et al., 1999c). This retroposon may therefore represent a family that has been active in recent human evolution. Here we report retroposon family on the $\mathrm{X}$ chromosome and phylogenetic analysis with those of other chromosomes.

\section{MATERIALS AND METHODS}

Sample DNA and PCR. A human monochromosomal somatic cell hybrid DNA panel was purchased from the Coriell Cell Repositories (Coriell Institute, USA). The X chromosomal panel sample was subjected to PCR amplification. SINE-R retroposons are amplified by the primer pair JS902 (5'-GAGAATGGGCCATGATGAC-3', bases 4-22) and JS903 (5'-GATCATTCTTGGATGTTTCT3', bases 466-485) from schizophrenic brain S11 cDNA (GenBank, accession no. AA772777) which has a high degree of sequence homology with SINE-R.C2, 11, 14, 19, HS307/HS408 and the long terminal repeats of human endogenous retrovirus HERV-K10 (Ono et al., 1987; Kim et al., 1999b). The PCR conditions followed were those of Kim et al. (1996) with an annealing temperature of $56^{\circ} \mathrm{C}$.

Cloning of PCR products. PCR products were separated on a $1.8 \%$ agarose gel, purified with the QIAEX II gel extraction kit (Qiagen) and cloned into the T-khs307 vector (Kim et al., 1998). Plasmid DNA was isolated by the alkali lysis method using the High Pure plasmid isolation kit (Boehringer Mannheim).

DNA sequencing and data analyses. Individual plasmid DNAs were screened for inserts by PCR. Positive samples were subjected to sequence analysis on both strands with T7 and M13 reverse primers using an automated DNA sequencer (Model 373A) and the DyeDeoxy terminator kit (Applied Biosystem). When the sequences of both strands were different, we did not include the sequences for analyses. SINE-R.C2 (L09706), SINE-R11 (X07417), SINE-R14 (X07418), SINE-R19 (X07419), FMR1 (L29074), HS408 (AB016782), HS307 (AB016781), SCHIZO (AA772777), 7-3 (AB025299), 7-11 (AB025399), 7-13 (AB025301), 7-14 (AB025302), 7-15 (AB025303), 17-1 (AB025304), 17-2 (AB025305), 17-11 (AB025305), 17-11 (AB025306), 17-12 (AB025307), 17-14 (AB025308), Y-2 (AB025287), Y-3 (AB025288), Y-4 (AB025289), Y-7 (AB025290), Y-8 (AB025291), Y-11 (AB025292), Y-13 (AB025293), Y-15 (AB025294), Y-16 (AB025295), Y-17 (AB025296), Y-18 (AB025297), Y-19 (AB025298), Ch-M1 (AB023860), Ch-M8 (AB023861), Ch-M9 (AB023862), Ch-M10 (AB023863), Ch-M11 (AB023864), Ch-M12 (AB023865), Ch-M13 (AB023866), Ch-M14 (AB023867), Ch-M15 (AB023868), Ch-M16 (AB023869), Ch-M17 (AB023870), Ch-M18 (AB023871), Ch-M19 (AB023872), Ch-M20 (AB023873), Go-M2 (AB023820), Go-M4 (AB023821), Go-M6 (AB023822), Go-M8 (AB023823), Go-M12 (AB023824), Go-M13 (AB023825) sequences were taken from the GenBank database. Sequence analyses were done with the aid of GAP, PILEUP, and PRETTY from the GCG program (Unicersity of Wisconsin). The neighborjoining phylogenetic analysis (Saitou and Nei, 1987) was performed with the MEGA program (Kumar et al., 1993). Statistical significance evaluation of the branching pattern was performed with 100 replications.

\section{RESULTS AND DISCUSSION}

SINE-R retroposons derived from the long terminal repeat (LTR) of the HERV-K family (Ono et al., 1987; Zhu et al., 1994) have been found to be hominoid-specific by PCR and sequencing analyses (Kim et al., 1999a,b,c). Comparing nucleotide sequences between SINE-R retroposons and HERV-K10 LTR elements, the SINE-R retroposons were found to be deleted by $367 \mathrm{bp}$, including the promoter region that precedes a polyadenylation signal, from the HERV-K10 LTR sequences (Fig. 1). We identified and sequenced 25 different SINE-R retroposons on the human X chromosome. These sequences are compared to the element (SINE-R.C2) originally identified in the $\mathrm{C} 2$ complement gene that is human specific (Zhu et al., 1994), to those elements (SINE-R11, R14, and R19) earlier reported in the human genome (Ono et al., 1987) and present also in hominoid primates (Kim et al., 1999a,b,c), to an element identified in the fragile $\mathrm{X}$ mental retardation gene (FMR-1), and to the elements we have identified on the 7, 17, and Y chromosomes (Kim and Crow, 1999, 2000) and in cDNA (SCHIZO) from postmortem brain. Also included are two elements (HS307 


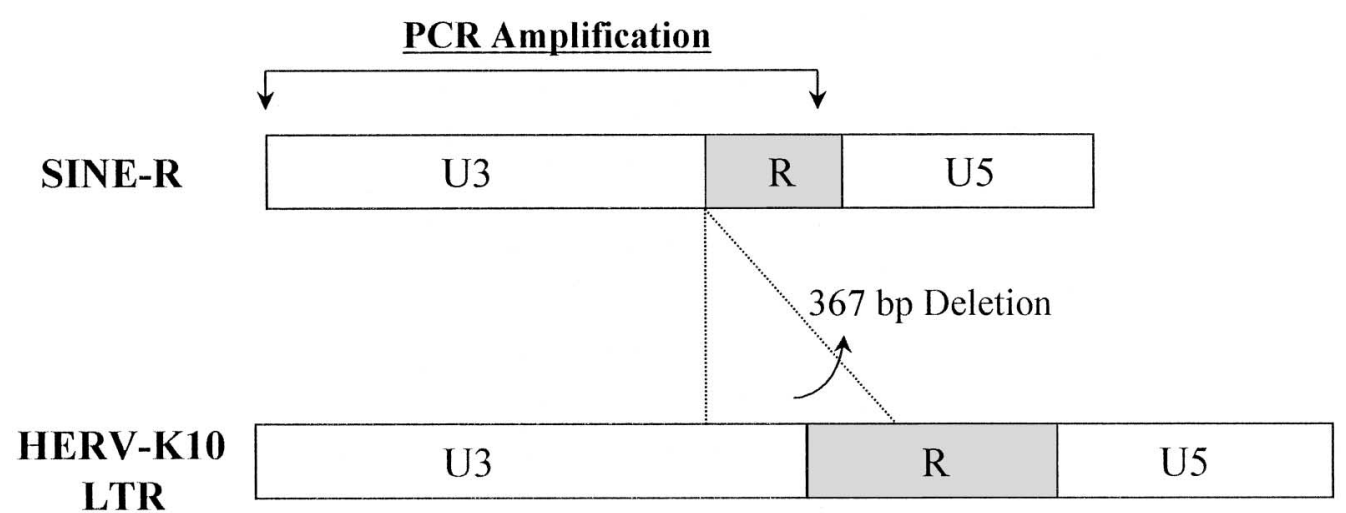

Fig. 1. Structural comparison of SINE-R retroposon and HERV-K10 LTR. Area of PCR amplification is indicated.

and 408) already located (Kim et al., 1999b) in the Xq21.3 region of homology to Yp (Sargent et al., 1996) that was generated after the separation of the chimpanzee and human lineages by a retroposition from the $\mathrm{X}$ to the $\mathrm{Y}$ chromosomes.

In order to understand the evolutionary relationships among retroposon family, we analysed all retroposons identified on chromosome 7, 17, and Y phylogenetically together with apes retroposons identified in our previous study (Kim and Crow, 1999, 2000; Kim et al., 1999a,b,c). As shown in Fig. 2, two features are illustrated. First, the pattern of phylogeny suggests an evolutionary "cascade" (retroposon derived from the HERV-K LTR element has been evolved randomly) rather than the separation of distinct groups. Second, within this cascade the set of elements that is furthest removed from the origin in the HERV-K10 sequence includes the SINE-R.C2 element, the HS307 sequence and a small group of elements (X-5, X-6, $\mathrm{X}-11, \mathrm{X}-13, \mathrm{X}-15, \mathrm{X}-22$, X-26, X-27, X-29) on the X chromosome with greater than $95 \%$ homology to these sequences. It appears possible that these are the most recently evolved elements in the class. Medstrand and Mager (1998) identified a phylogenetic cluster (their cluster 9) of HERV-K LTRs that are specific to the human genome; it appears that the SINE-R.C2 cluster including Ch-M10, 16, 20, Go-M6, 13 and X-6, 22, 27 may have a similar status. Clustering of mobile elements that transpose via an RNA intermediate is unexpected, since there is no reason to suspect that progeny elements would preferentially transpose to the chromosome of the parent. These phenomena allow us to speculate that they arise from unequal recombination as tandemly duplicated elements. It appears reasonable to explain that some lineages of the SINE-R retroposon family have evolved independently during primate evolution in the phylogenetic analysis between the human and African great apes. Such evolutionary pattern has been detected in HERV-K LTR and LTR-like elements (Medstrand and Mager, 1998; Kim et al., 1999d).

Because the sex chromosomes are subject to relatively rapid evolutionary change, location of mobile elements yields a further opportunity to map the time course of retroposition. Besides the pseudoautosomal regions, within which there is recombination in male meiosis, and therefore strict homology of sequences on the $X$ with those on the Y, a number of blocks of X-Y homology have been identified (Affara et al., 1996; Lambson et al., 1992) and their origins in primate evolution, often by translocation from the $\mathrm{X}$ to the $\mathrm{Y}$ chromosome, determined. It will be interesting to examine whether the HS307 and HS408 sequences that we have identified within the Xq21.3 region (Kim et al., 1999b) are present within the block of homology in Yp that was generated after the separation of the chimpanzee and human lineages (Sargent et al., 1996).

There is a further interest of the sex chromosomes in relation to events of potential evolutionary significance. One explanation of Haldane's rule that when in a species cross one sex is infertile or inviable it is the hetero-gametic sex is that the gene or genes involved in speciation are preferentially located on the sex chromosomes (Coyne and Orr, 1998). Moreover it has been suggested that the processes of sexual selection and speciation are related (Kaneshiro, 1980) and sex-linked genes could be relevant to this relationship (Civetta and Singh, 1998; Ford and Aquadro, 1996). It has further been argued (Crow, 1998) that a relationship between sexual selection and speciation has relevance to Homo sapiens in that there is evidence that a gene in the $\mathrm{X}-\mathrm{Y}$ homologous class played a role in the process of cerebral hemispheric differentiation that may underlie the human capacity for language (Crow et al., 1998). Thus further investigation of the phylogenies of mobile elements that have been active in hominoid primates, their locations on the sex chromosomes, and relationships to chromosomal change, may cast light on the origin of the species.

An element apparently identical in sequence to SINER.C2 has recently been reported as the cause of Fukuyama-type muscular dystrophy (Kobayashi et al., 1998), and this appears to be the first association of this class of elements with disease. SINE-R elements may also influence gene expression. In addition to the sequence we 


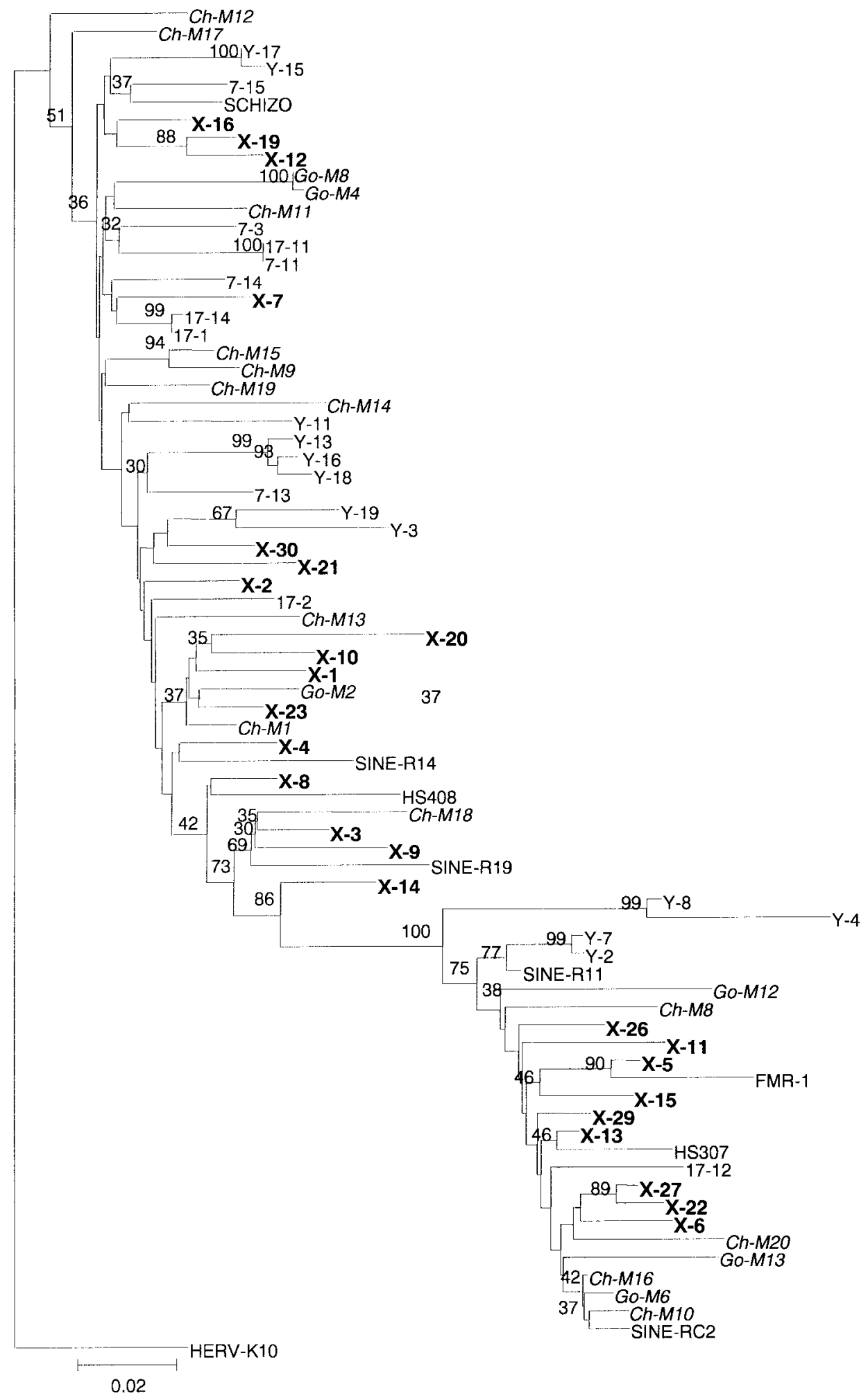

Fig. 2. Phylogenetic tree obtained by neighbor-joining method for the retroposon family. Statistical robustness was tested by bootstrap analysis. The numbers to the left of the nodes of the tree branches represent the number of times out of 100 trees. HERV-K10 was used as outgroup. The nucleotide sequence data reported in this paper will be appear in the DDBJ/EMBL/GenBank nucleotide sequence databases with the accession numbers AB023687 (X-1), AB023688 (X-2), AB023689 (X-3), AB023690 (X-4), AB023691 (X-5), AB023692 (X-6), AB023693 (X-7), AB023694 (X-8), AB023695 (X-9), AB023696 (X-10), AB023697 (X-11), AB023698 (X-12), AB023699 (X13), AB023700 (X-14), AB023701 (X-15), AB023702 (X-16), AB023703 (X-19), AB023704 (X-20), AB023705 (X-21), AB023706 (X-22), AB023707 (X-23), AB023708 (X-26), AB023709 (X-27), AB023710 (X-29), AB023711 (X-30). 
observed in cDNA from post-mortem brain, an instance in which a SINE element acquired a role in signal transduction in the course of mammalian evolution has also been described (Shimamura et al., 1998). The phylogeny of SINE-R elements may contribute to an understanding of the role of such events in human evolution.

In summary, SINE-R elements, derivatives of the human endogenous retrovirus K10 (HERV-K10), are present on the human $\mathrm{X}$ chromosome, and the range of sequence variation resembles that already found in representatives of this class of retroposons on chromosomes 7, 17, $\mathrm{Y}$ and in African great apes. Those elements have evolved independently during primate evolution. The SINE-R.C2 element, which was first described in the $\mathrm{C} 2$ complement gene, and so far has been found only in the human genome, is a member of a group of SINE-R elements that have evolved furthest from the parent HERV-K10 sequence. The location of SINE-R elements (such as HS307 and HS408) within regions of $\mathrm{X} / \mathrm{Y}$ homology that have arisen by translocations that can be dated in the course of hominoid evolution provides a window on the possible role of such elements in genome change.

This work was supported by Pusan National University Research Grant 1999 (Korea) and Medical Research Council (UK).

\section{REFERENCES}

Affara, N., Bishop, C., Brown, W., Cooke, H., Davey, P., Ellis, N., Graves, J. M., Jones, M., Mitchell, M., Rappold, G., TylerSmith, C., Yen, P., and Lau, Y.-F. C. (1996) Report of the second international workshop on Y chromosome mapping 1995. Cytogenet. Cell Genet. 73, 33-76.

Baltimore, D. (1985) Retroviruses and retrotransposons: the role of reverse transcription in shaping the eukaryotic genome. Cell 40, 481-482.

Brosius, J., and Tiedge, H. (1995) Reverse transcriptase: mediator of genomic plasticity. Virus Genes 11, 163-179.

Civetta, A., and Singh, R. S. (1998) Sex-related genes, directional sexual selection, and speciation. Mol. Biol. Evol. 15, 901909.

Coyne, J. A., and Orr, H. A. (1998) The evolutionary genetics of speciation. Philosophical Transactions of the Royal Society, London Series B 353, 287-309.

Crow, T. J. (1998) Sexual selection, timing and the descent of Man: a genetic theory of the evolution of language. Curr. Psychol. Cognit. 17, 1079-1114.

Crow, T. J., Crow, L. R., Done, D. J., and Leask, S. J. (1998) Relative hand skill predicts academic ability: global deficits at the point of hemispheric indecision. Neuropsychologia 36, 12751282.

Ford, M. J., and Aquadro, C. F. (1996) Selection on X-linked genes during speciation in the Drosophila athabasca complex. Genetics 144, 689-703.

Ginzburg, L. R., Bingham, P. M., and Yoo, S. (1984) On the theory of speciation induced by transposable elements. Genetics 107, 331-341.

Izvak, Z., Ivics, Z., Garcia-Estefania, D., Fahrenkrug, S. C., and Hackett, P. B. (1996) DANA elements: a family of composite, tRNA-derived short-interspersed DNA elements associated with mutational activities in zebrafish (Danio rerio). Proc. Natl. Acad. Sci. USA 93, 1077-1081.

Kaneshiro, K. Y. (1980) Sexual isolation, speciation and the direction of evolution. Evolution 34, 437-444.

Kazazian, H. H., and Moran, J. V. (1998) The impact of L1 retrotransposons on the human genome. Nature Genet. 19, 19-24.

Kido, Y., Aono, M., Yamaki, T., Matsumoto, K., Murata, S., Saneyoshi, S., and Okada, N. (1991) Shaping and reshaping of samonid genomes by amplification of rRNA-derived retroposons during evolution. Proc. Natl. Acad. Sci. USA 88, 2236-2330.

Kim, H.-S., Chen, Y., and Lonai, P. (1998) Complex regulation of multiful cytohesin-like genes in murine tissues and cells. FEBS Lett. 433, 312-316.

Kim, H.-S., and Crow, T. J. (1999) Presence and phylogenetic relationships of a hominoid-specific retroposon family on the human Y chromosome. Zool. Sci. 16, 963-970.

Kim, H.-S., and Crow, T. J. (2000) Phylogenetic relationships of a class of hominoid-specific retro-elements (SINE-R) on human chromosomes 7 and 17. Ann. Hum. Biol. 27, 83-93.

Kim, H.-S., Hirai, H., and Takenaka, O. (1996) Molecular features of the TSPY gene of gibbons and Old World monkeys. Chrom. Res. 4, 500-506.

Kim, H.-S., Takenaka, O., and Crow, T. J. (1999a). Cloning and nucleotide sequence of retroposons specific to hominoid primates derived from an endogenous retrovirus (HERVK). AIDS Res. Hum. Retroviruses 15, 595-601.

Kim, H.-S., Wadekar, R. V., Takenaka, O., Winstanley, C., Mitsunaga, F., Kageyama, T., Hyun, B.-H., and Crow, T. J. (1999b). SINE-R.C2 (a Homo Sapiens specific retroposon) is homologous to cDNA from post-mortem brain in schizophrenia and to two loci in the Xq21.3/Yp block linked to handedness and psychosis. Am. J. Med. Genet. (Neuropsychiat. Genet.) 88, 560-566.

Kim, H.-S., Wadekar, R. V., Takenaka, O., Hyun, B.-H., and Crow, T. J. (1999c). Phylogenetic analysis of a retroposon family in African great apes. J. Mol. Evol. 49, 699-702.

Kim, H.-S., Wadekar, R. V., Takenaka, O., Hyun, B.-H., and Crow, T. J. (1999d). Phylogenetic analysis of HERV-K LTR-like elements in primates: presence in some New World monkeys and evidence of recent parallel evolution in these species and in Homo sapiens. Arch. Virol. 144, 2035-2040.

Kobayashi, K., Nakahori, Y., Miyaki, M., Matsumura, K., Kondolida, E., Nomura, Y., Segawa, M., Yoshioka, M., Saito, K., Osawa, M., Hamano, K., Sakakihara, Y., Nonaka, I., Nakagome, Y., Kanazawa, I., Nakamura, Y., Tokunaga, K., and Toda, T. (1998) An ancient retrotransposal insertion causes Fukuyama-type congenital muscular dystrophy. Nature 394, 388-392.

Kumar, S., Tamura, K., and Nei, M. (1993) MEGA:molecular evolutionary genetics analysis, version 1.01. Pennsylvania State University, University Park, PA 16802, USA.

Lambson, B., Affara, N. A., Mitchell, M., and Ferguson-Smith, M. A. (1992). Evolution of DNA sequence homologies between the sex chromosomes in primate species. Genomics 14, $1032-1040$

Medstand, P., and Mager, D. L. (1998) Human-specific integrations of the HERV-K endogenous retrovirus family. J. Virol. 72, 9782-9787.

McClintock, B. (1984) The significance of responses of the genome to challenge. Science 226, 792-801.

Miki, Y. (1998). Retrotransposal integration of mobile genetic elements in human diseases. J. Hum. Genet. 43, 77-84.

Okada, N. (1991). SINEs: short interspersed repeated elements 
of the eukaroyotic genome. Trends Ecol. Evol. 6, 358-361.

Okada, N., Hamada, M., Ogiwara, I., and Ohshima, K. (1997) SINEs and LINEs share common 3' sequences: a review. Gene 205, 229-243.

Ono, M., Kawakami, M., and Takezawa, T. (1987) A novel human nonviral retroposon derived from an endogenous retrovirus. Nucleic Acids Res. 21, 8725-8737.

Rose, R. R., and Doolittle, W. F. (1983) Molecular mechanisms of speciation. Science 220, 157-162.

Saitou, N., and Nei, M. (1987) The neighbor-joining method: a new method for reconstructing phylogenetic trees. Mol. Biol. Evol. 4, 406-425.

Sargent, C. A., Briggs, H., Chalmers, I. J., Lambson, B., Walker, E., and Affara, N. A. (1996) The sequence organization of Yp/ proximal Xq homologous regions of the human sex chromosomes is highly conserved. Genomics 32, 200-209.

Shimamura, M., Nikaido, M., Ohshima, K., and Okada, M. (1998)
A SINE that acquired a role in signal transduction during evolution. Mol. Biol. Evol. 15, 923-925.

Shimamura, M., Yasue, H., Ohshima, K., Abe, H., Kato, H., Kishiro, T., Goto, M., Munechika, I., and Okada, N. (1997) Molecular evidence from retroposons that whales form a clade within even-toed ungulates. Nature 338, 666-670.

Smit, A. F. A. (1996) The origin of interspersed repeats in the human genome. Curr. Opin. Genet. Dev. 6, 743-748.

Sverdlov, E. D. (1998) Perpetually mobile footprints of ancient infections in the human genome. FEBS Lett. 428, 1-6.

Terai, Y., Takahashi, K., and Okada, N. (1998) SINE cousins: the 3 -end tails of the two oldest and sitantly related families of SINEs are descended from the 3 ' ends of LINEs with the same genealogical origin. Mol. Biol. Evol. 15, 1460-1471.

Zhu, Z.-B., Jian, B., and Volanakis, J. E. (1994) Ancestry of SINER.C2 a human specific retroposon. Hum. Genet. 93, 545551. 